\title{
Magnetofection Potentiates Gene Delivery to Cultured Endothelial Cells
}

\author{
Florian Krötz ${ }^{a}$ Hae-Young Sohn ${ }^{b}$ Torsten Gloe ${ }^{a}$ Christian Plank ${ }^{c}$ \\ Ulrich Pohla \\ a Institute of Physiology and ${ }^{b}$ Cardiology Division, Medizinische Poliklinik - Innenstadt, \\ Ludwig Maximilian University and 'Department of Experimental Oncology, Technical University, Munich, Germany
}

\author{
Key Words \\ Endothelial cells · Magnetofection · Plasmid vectors
}

\begin{abstract}
Modification of cellular functions by overexpression of genes is increasingly practised for research of signalling pathways, but restricted by limitations of low efficiency. We investigated whether the novel technique of magnetofection (MF) could enhance gene transfer to cultured primary endothelial cells. MF of human umbilical vein endothelial cells (HUVEC) increased transfection efficiency of a luciferase reporter gene up to 360-fold compared to various conventional transfection systems. In contrast, there was only an up to 1.6-fold increase in toxicity caused by MF suggesting that the advantages of MF outbalanced the increase in toxicity. MF efficiently increased transfection efficiency using several commercially available cationic lipid transfection reagents and polyethyleneimine (PEI). Using PEI, even confluent HUVEC could be efficiently transfected to express luciferase activity. Using a green fluorescent protein vector maximum percentages of transfected cells amounted up to $38.7 \%$ while PEI without MF resulted in only $1.3 \%$ transfected cells. Likewise, in porcine aortic endothelial cells MF increased expression of a luciferase or a $\beta$-galactosidase reporter, reaching an efficiency of $37.5 \%$ of cells. MF is
\end{abstract}

an effective tool for pDNA transfection of endothelial cells allowing high efficiencies. It may be of great use for investigating protein function in cell culture experiments.

Copyright $@$ @ 2003 S. Karger AG, Basel

\section{Introduction}

Numerous modifications of gene delivery techniques have been used to improve the efficiency of gene expression in mammalian cells [1-3]. High transfection efficiency is important in approaches, where transfected gene overexpression is used as a tool to study gene/protein function as well as in therapeutic applications. Among the tissues targeted, the vascular endothelium has a key role, as treatment of endothelial dysfunction by gene transfer is assumed to prevent vascular diseases such as hypertension, atherosclerosis, vascular thrombotic disease and their complications [3-5]. Despite intensive efforts to optimize gene delivery to endothelial cells of human and other species [6-10], so far only low efficiencies of target gene expression have been reported [7-9].

We have recently shown in several cell lines that coupling of magnetic nanoparticles to gene vectors of any kind results in a dramatic increase of the uptake of these vectors and consequently, target protein expression

\begin{tabular}{ll}
\hline KARGER & ( ) 2003 S. Karger AG, Basel \\
1018-1172/03/0405-0425\$19.50/0 \\
$\begin{array}{l}\text { Fax+4161306 12 34 } \\
\text { www.karger.com }\end{array}$ & $\begin{array}{l}\text { Accessible online at: } \\
\text { www.karger.com/jvr }\end{array}$
\end{tabular}

Dr. Florian Krötz

Institute of Physiology, Ludwigs-Maximilians-Universität

Schillerstrasse 44

DE-80336 München (Germany)

Tel. +49 895996 384, Fax +49 895996 378, E-Mail fkroetz@lmu.de 
[11]. Moreover, magnetofection (MF) allowed locally enhanced expression of $\beta$-galactosidase activity in several cell lines [11] and rapid and efficient transfer of small nucleic acids into endothelial cells at low toxicity [12]. However, so far it has not been investigated whether this technique could also succeed in enhancing efficiency of nonviral vector-mediated gene expression in primary cells like endothelial cells, which are well-known to be virtually resistant to efficient transfection with pDNA [7-9]. If this could be performed, a promising technique for in vivo vascular gene delivery and a new tool for efficient research would be at hand, which also could potentially overcome many of the problems of in vivo gene transfer. We, therefore, investigated, whether MF could be a feasible technique to increase the efficiency of gene transfer into primary vascular cells like endothelial cells and whether this influences cytotoxicity of transfections.

\section{Methods}

\section{Endothelial Cell Culture}

Human umbilical vein endothelial cells (HUVEC) were prepared and grown to confluence in medium 199 (Sigma, Germany) supplemented with 20\% newborn calf serum (NBCS, Biomol, Germany) and $20 \%$ endothelial growth medium (Promocell, Germany) as previously described [13]. In addition, fresh porcine aortae were obtained from the local slaughterhouse. Cells were harvested from aortae as described [14] and grown to confluence in DMEM supplemented with glutamine $(200 \mathrm{mM})$, penicillin/streptomycin and $20 \%$ fetal calf serum (FCS, Biomol). Only cells of the 1st to 3rd subpassage were used for experiments.

\section{Transfection of pDNA}

On the day before MF, 5,000 or 10,000 HUVEC or porcine aortic endothelial cells (PAEC) in a volume of $100 \mu \mathrm{l}$ were seeded into each well of a flat-bottom 96-well dish, to obtain cell layers of 50 or 90-100\% confluence, respectively. To reach confluence states of $50 \%$ on the following day, 5,000 cells/well proved to be sufficient, whilst seeding of 10,000 cells/well usually resulted in confluence states of $95 \%$. In some experiments, to reach highly confluent cell layers, 50,000 cells/well were plated onto the cell culture dishes. Before MF, medium was removed and $50 \mu \mathrm{l} /$ well of serum-free DMEM was added. For preparation of the MF mixture the desired amount of pDNA was mixed with DMEM followed by the addition of CombiMAG and gentle mixing by pipetting up and down 5 times (solution 1). The CombiMAG to pDNA ratio was $1: 1$ for liposomal transfection reagents and 2:1 for polyethyleneimine (PEI):DNA. For controls, DMEM was added as sham solution instead of CombiMAG. Then the conventional transfection reagents or PEI were mixed with various amounts of DMEM (solution 2), to achieve the pDNA-transfection ratios recommended by the manufacturers. PEI $(25 \mathrm{kD})$ was added to a PEI:DNA N/P ratio of 8 . Solution 1 and 2 were mixed and incubated at room temperature for $30 \mathrm{~min}$. Then $50 \mu \mathrm{l}$ of each mixture were added per well, resulting in the desired final concentration of $\mathrm{pDNA} / \mathrm{well}$. Subse- quently, the cell culture plates were placed on Nd-Fe-B magnetic plates (Chemicell, Berlin, Germany) for 15 min. After another $4 \mathrm{~h}$ of incubation, medium was removed and new medium added. Twenty-four hours after MF, the respective assays for detection of reporter expression were performed. Plasmids used were p55pCMV-IVS-luc ${ }^{+}$(firefly luciferase), EF $\alpha$ GFP (green fluorescent protein), and pCMV- $\beta$-gal [11].

\section{Luciferase Activity}

Twenty-four hours after transfection, cells were washed once with PBS and then incubated with $100 \mu \mathrm{l}$ of lysis buffer $(0.1 \%$ Triton X100 in $250 \mathrm{~m} M$ Tris, $\mathrm{pH} 7.8$ ). 10-50 $\mu 1$ of each cell lysate were transferred to black 96-well plates, mixed with $100 \mu \mathrm{l}$ of luciferin buffer [60 $\mathrm{m} M$ dithiothreitol, $10 \mathrm{~m} M$ magnesium sulfate, $1 \mathrm{~m} M$ ATP, $30 M$ $D(-)$-luciferin, in $25 \mathrm{~m} M$ glycyl-glycine, $\mathrm{pH}$ 7.8] and assayed for bioluminescence using a TopCount instrument (Canberra Packard, Groningen, The Netherlands). The protein content of the cell lysates was determined using the BioRad protein assay adapted for use in a 96-well plate format. Specific luciferase activity in nanograms luciferase per milligram of protein was calculated by a calibration curve, which was obtained from luminescence of a serial dilution of luciferase (Roche, Mannheim, Germany).

\section{Flow Cytometry}

The efficiency of gene transfer of GFP was analyzed by flow cytometry. Cells were washed once in PBS (containing calcium), lysed by the addition of a trypsin solution for $2 \mathrm{~min}$, followed by the addition of PBS (without calcium) for another 2 min and fixation in formalin (final concentration 2\%). To assess cell viability in flowcytometric evaluations, cells were exposed to propidium iodide (PI; $500 \mathrm{ng} / \mathrm{ml}$ ) for $30 \mathrm{~min}$ before harvesting and analyzing them. Fluorescence intensity was analyzed using a FACScan flow cytometer (Becton Dickinson). Data were analyzed using the CellQuest (Becton Dickinson) software. Transfection efficiency was expressed as percent of viable cells positively stained with GFP.

\section{$\beta$-Galactosidase Staining}

For X-gal staining, cells were washed twice in PBS, fixed in 2\% formalin $(\mathrm{w} / \mathrm{v})$ solution and stained by addition of a solution containing $400 \mathrm{~m} M$ potassium ferricyanide, $400 \mathrm{~m} M$ potassium ferrocyanide, $200 \mathrm{~m} M$ magnesium chloride and $1 \mathrm{mg} / \mathrm{ml} \mathrm{X}$-gal (Eurobio, Les Ulis, France) for $1 \mathrm{~h}$.

\section{Toxicity}

Toxicity was measured as LDH release using a CytoTox $96^{\circledR}$ assay (Promega, Madison, Wisc., USA) following the manufacturer's instructions. Cells were treated as indicated and $50 \mu \mathrm{l}$ of supernatants mixed with $50 \mu$ of the assay's substrate mix and incubated at room temperature for $30 \mathrm{~min}$. The reaction was stopped by adding $50 \mu \mathrm{l}$ of acetic acid $(1 M)$ and absorption measured at $486 \mathrm{~nm}$. For positive controls, cells were treated with $1 \%$ Triton X-100 in Tyrode's buffer for $10 \mathrm{~min}$. To assess the number of dead cells after this treatment, trypan blue staining was performed. Maximum toxicity was set as the LDH release caused by Triton X-100 (1\%) treatment, while LDH release from control cells was assumed not to be due to toxic causes. The LDH release caused by the transfections then yielded a percentage of the toxicity caused by Triton treatment. LDH activity after MF is expressed as $n$-fold increase over standard transfection. Accordingly, the increase in toxicity caused by MF (table 1) in comparison to standard transfection is the ratio of toxicity caused by MF 
Fig. 1. MF increases luciferase expression in cultured HUVEC. Transfected luciferase activity was increased by MF using different commercially available transfection reagents $(\mathbf{a}, \mathbf{c}, \mathbf{d})$ or DNA coupled to PEI (b). Vectors were complexed to transMAG ${ }^{\mathrm{PEI}}$ by electrostatic interaction and salt-induced colloid aggregation followed by incubation with the indicated transfection reagents or PEI. Maximum luciferase activity of nonconfluent cells (confluence state: 50-75\%) was obtained by MF using FuGENE with 500 ng of DNA $(\mathbf{a}, \mathrm{n}=6)$. High efficiencies were also reached for PEI $(\mathbf{b}, \mathrm{n}=6)$. Throughout most DNA concentrations and different nonviral transfection reagents (a: FuGENE, c: Lipofectamine, $\mathrm{n}=6$, d: DMRIE, $\mathrm{n}=6$ ) $\mathrm{MF}(\boldsymbol{\square})$ was superior to conventional transfection ( $\square$ ). Numbers above bars represent fold increase achieved by MF compared to conventional nonviral transfection techniques.
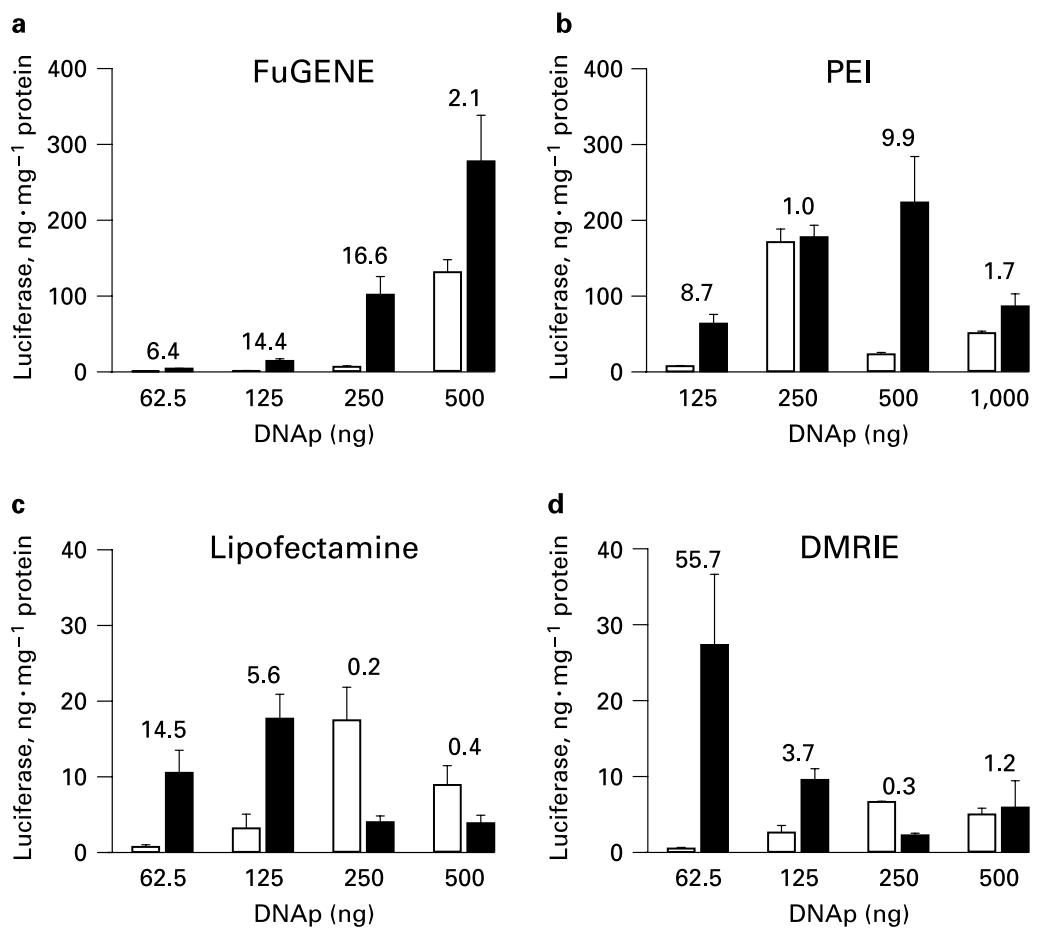

and the toxicity of standard transfection. The increase in efficiency by MF corrected for toxicity (table 1) is the ratio of increase in efficiency and increase in toxicity caused by MF and is expressed as n-fold.

\section{Materials}

Magnets and magnetic beads (CombiMAG) were from Chemicell (Berlin, Germany). PEI (25 kD) was from Aldrich (Deisenhofen, Germany), the Luciferase assay kit was purchased from Promega (Germany) and 5-bromo-4-chloro-3-indolyl- $\beta$ - $D$-galactopyranoside (X-gal) was from Biotech Trade \& Service (Germany).

\section{Statistics}

All data are expressed as means \pm standard deviation. The Student's $t$ test was performed for all comparisons. For paired observations the paired $t$ test was performed. An error probability level of $\mathrm{p}<0.05$ was considered significant.

\section{Results}

\section{Transfection of pDNA into Subconfluent HUVEC}

Several commercially available transfection reagents were tested for MF compared with standard transfection techniques. The vectors contained genes either encoding luciferase $\left(\mathrm{Luc}^{+}\right)$, GFP or $\beta$-galactosidase ( $\beta$-gal). In addition to commercially available transfection reagents (Li-
Table 1. Transfection efficiency of a $\beta$-galactosidase plasmid in PAEC

\begin{tabular}{|c|c|c|c|c|}
\hline \multirow{2}{*}{$\begin{array}{l}\text { pDNA } \\
\text { ng }\end{array}$} & \multicolumn{2}{|l|}{ FuGENE } & \multicolumn{2}{|l|}{ PEI } \\
\hline & $\begin{array}{l}\text { standard TF } \\
\% \text { of cells }\end{array}$ & $\begin{array}{l}\text { MF } \\
\% \text { of cells }\end{array}$ & $\begin{array}{l}\text { standard TF } \\
\% \text { of cells }\end{array}$ & $\begin{array}{l}\text { MF } \\
\% \text { of cells }\end{array}$ \\
\hline 62.5 & $9 \pm 5$ & $30.1 \pm 7^{*}$ & - & - \\
\hline 125 & $17.6 \pm 8$ & $24.9 \pm 12$ & $1.2 \pm 1$ & $4.3 \pm 4^{* *}$ \\
\hline 250 & $18.6 \pm 7$ & $36.6 \pm 13^{*}$ & $3.9 \pm 3$ & $6.7 \pm 4^{* *}$ \\
\hline 500 & $19.6 \pm 6$ & $20.1 \pm 7$ & $5.8 \pm 3$ & $14.7 \pm 5^{* *}$ \\
\hline 1,000 & - & - & $6.3 \pm 5$ & $37.5 \pm 7 * *$ \\
\hline
\end{tabular}

Percentages of cells (PAEC) staining positive for X-gal are shown. Data representative of means \pm SD of 6 independent experiments, $* \mathrm{p}>0.05,{ }^{* *} \mathrm{p}<0.01$ vs. standard transfection. TF $=$ Transfection. pofectamine $^{\mathrm{TM}}$, FuGENE ${ }^{\mathrm{TM}}$, DMRIE-C ${ }^{\mathrm{TM}}$ ) pDNA complexed with PEI was transfected. In all experiments transfection conditions were identical except for the presence or absence of magnetic beads.

Transfection efficiencies were assessed over a range of different concentrations. Figure 1 summarizes mean effi- 
Fig. 2. MF enables gene transfer to confluent HUVEC. Using MF, confluent cells (confluence state 90-100\%) exhibited high expression of luciferase activity. Such MF resulted in the highest overall luciferase activity achieved independent of confluence state (PEI with 1,000 ng DNA, n = 6). Whereas conventional transfection did not result in relevant luciferase activity in confluent cells, several reagents such as PEI (a), Lipofectamine (b), DMRIE (c), or FuGENE (d), $(\mathrm{n}=6$ each) achieved notable levels of activity of the reporter gene in combination with MF. $\mathbf{\square}=$ MF; $\square=$ conventional transfection.

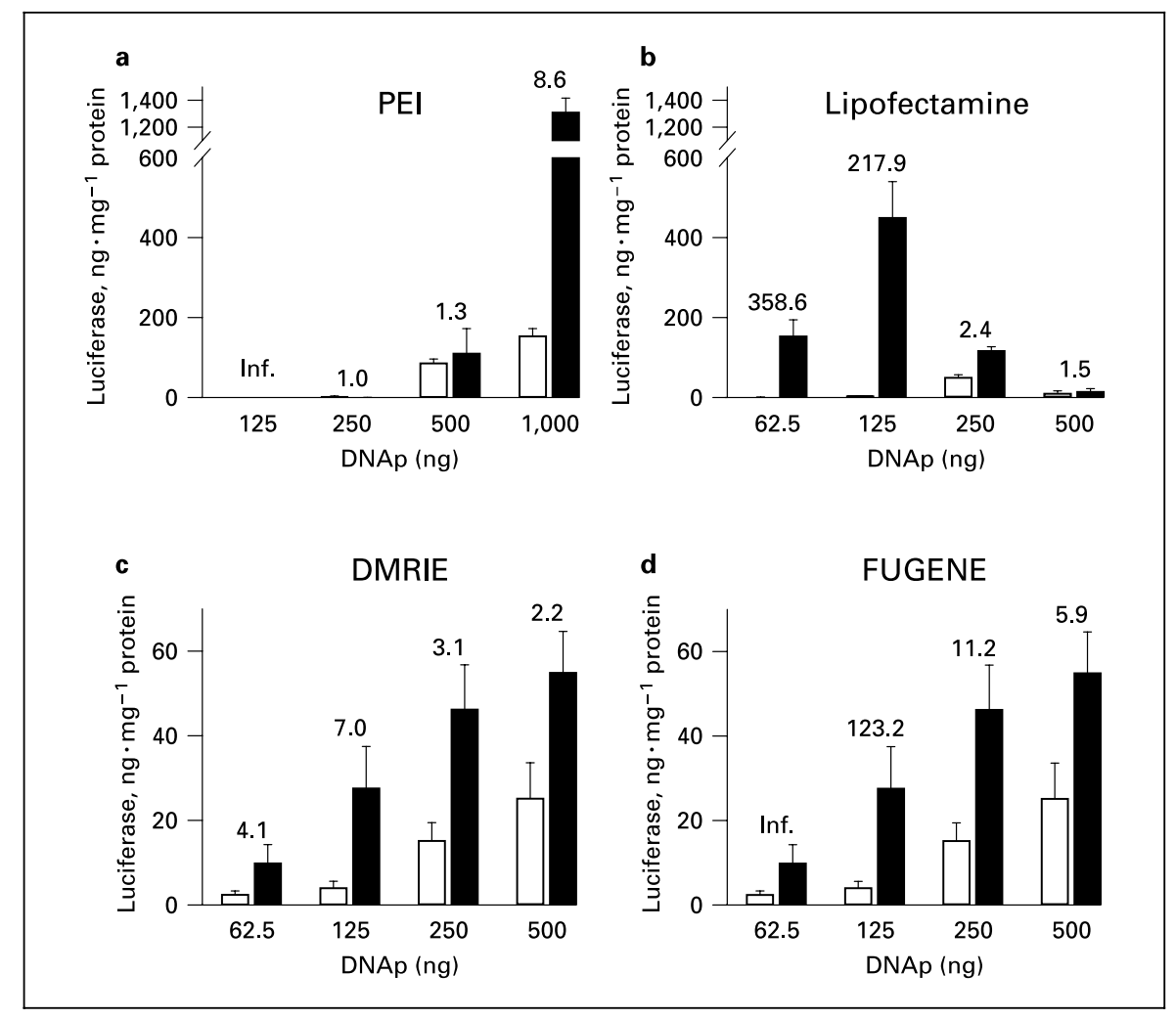

ciencies of diverse lipid transfection reagents and of PEI. The highest luciferase activity following transfection of a luc ${ }^{+}$-expressing plasmid was reached by MF of pDNA (at $500 \mathrm{ng} /$ well) in combination with FuGENE. MF in this setup was 2.1-fold more effective than the respective conventional transfection. The increases in efficiency, however, were only in part correlated with increasing pDNA doses. Furthermore, there was not a simple shift in dose dependency towards lower doses for the MF. In all cases, except one where the MF and the standard protocol accomplished a similar efficiency, maximum efficiencies were reached by a transfection procedure using MF. Furthermore, in many cases MF needed much lower doses of pDNA to reach comparable results than the conventional method. Similar results were obtained when using GenePorter $^{\mathrm{TM}}$ or Effectene ${ }^{\mathrm{TM}}$ reagents combined with MF with a luciferase plasmid (not shown).

\section{Transfection of pDNA into Confluent HUVEC}

We also tested the efficiency of the MF technique in cells that had just reached confluency and are, therefore, more suitable for designing experiments in which a more physiological state of the endothelium is desired or in which harvesting a certain amount of protein is planned (confluency state approximately 95\%), which are generally thought not to promote expression of transfected genes. Using conventional transfection, PEI yielded the highest luciferase activity using high amounts of pDNA (1,000 ng, $152 \pm 12 \mathrm{ng}$ luciferase/mg protein). Using CombiMAG the same concentration of pDNA reached a luciferase activity of 1,306 $\pm 69 \mathrm{ng}$ luciferase/mg protein, which not only represented an 8.6-fold increase of activity compared to the standard protocol, but also the highest overall luciferase activity reached in HUVEC irrespective of the confluence state or transfection reagent used $(n=6$, $p<0.01$, fig. 2a). Of the other conventional reagents used in confluent HUVEC, only Lipofectamine yielded a luciferase activity worth mentioning (250 ng pDNA, $50 \pm$ $5 \mathrm{ng}$ luciferase/mg protein). The same setup with MF reached an activity of $121 \pm 11 \mathrm{ng}$ luciferase/mg protein (2.4-fold increase, $n=6, p<0.01$ ). Even more was reached combining MF and Lipofectamine at pDNA levels of $125 \mathrm{ng} \mathrm{pDNA} /$ well $(469 \pm 92 \mathrm{ng}$ luciferase/mg protein, 217.9-fold increase vs. respective standard transfection, $\mathrm{n}=6, \mathrm{p}<0.01$, fig. $2 \mathrm{~b}$ ). Although FuGENE did not reach the same efficiency in confluent cells as in subconfluent HUVEC, in combination with MF it increased luciferase activity severalfold over the whole range of pDNA con- 
centrations tested (fig. $2 \mathrm{~d}, \mathrm{n}=6$ ). Similar results were obtained for DMRIE (fig. $2 \mathrm{c}, \mathrm{n}=6$ ).

The combination that had reached the highest luciferase activities for MF (1,000 ng pDNA and MF) was also tested for a plasmid coding for GFP. Using a flow-cytometric analysis after previous PI staining to exclude dead cells, a mean efficiency of $39 \pm 2 \%$ of living cells that stained positive for GFP could be obtained $(n=6$, counting a total of 5,000 cells/experiment, $\mathrm{p}<0.01$ ). Without magnetic beads only $1 \pm 0.4 \%$ were reached by the same setup. At $500 \mathrm{ng}$ of pDNA in combination with PEI with or without MF comparable differences were observed, although at lower absolute efficiencies (fig. 3a, representative pictures: $b-e)$. High efficiency, however, was reached at considerable rates of cytotoxicity regardless of whether the MF technique was used or not (33.4\% staining positive for PI when using MF vs. $28.7 \%$ without MF).

\section{Dependence of Transfection Efficiency on Confluency State of HUVEC}

To gain more insights into the optimal conditions for transfection of endothelial cells we next investigated the influence of the confluency state of the endothelial cells on transfection efficiency. To this end, we transfected equal amounts of pDNA (500 ng/well, see also above) of a GFPcoding plasmid to cells in five different confluency states. Cells were harvested and after plating them on new 24well dishes they were differently diluted so the confluency state on the day of transfection altered from high confluency (confluency reached on the same day as seeding the cells, after seeding 50,000 cells/well) across different levels of subconfluency $(95 \%, 10,000$ cells/well; 75\%, 8,000 cells/well; $60 \%, 6,000$ cells/well) to low confluency $(50 \%, 5,000$ cells/well). Flow-cytometric analysis of cells, $48 \mathrm{~h}$ after transfection, revealed a linear correlation between the confluency state and the level of GFP-expressing cells, which decreased with increasing confluency (fig. 3f). Cells at a low confluency state not only showed least sensitivity to the toxicity of the procedure $(24.3 \pm$ $1 \%$ ), but were best to transfect reaching efficiency levels of $16.5 \pm 0.5 \%$ compared to an efficiency of $4.0 \pm 0.1 \%$ for highly confluent cells $(n=4$ each, $\mathrm{p}<0.01)$. In addition, highly confluent cells also revealed the highest sensi-

Fig. 3. Efficiency of MF in HUVEC. a MF of a GFP-expressing vector achieved highest efficiency HUVEC when transMAGPEI-DNA complexes were coupled to PEI. Flow-cytometric analysis revealed maximum transfection efficiency of nearly $39 \%(\mathrm{n}=6, \square=$ conventional transfection, $\boldsymbol{\square}=$ MF, numbers above bars: efficiency of GFP

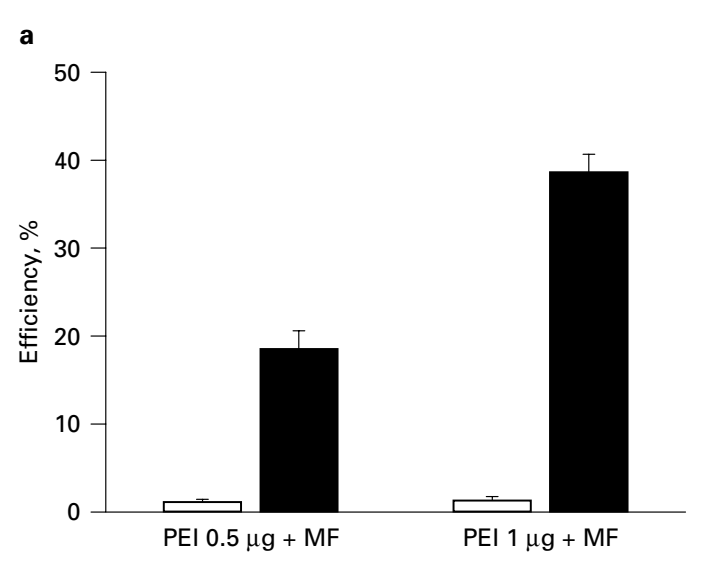

b
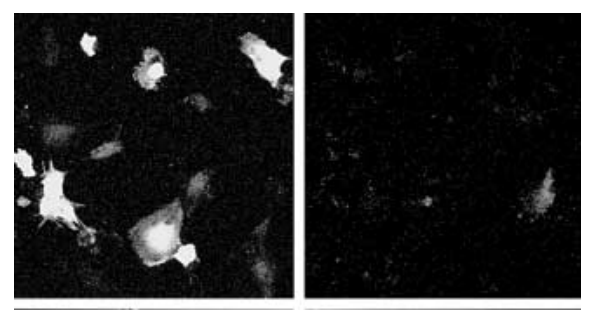

d

c

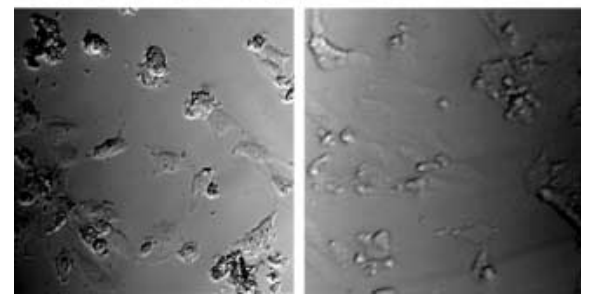

e

f

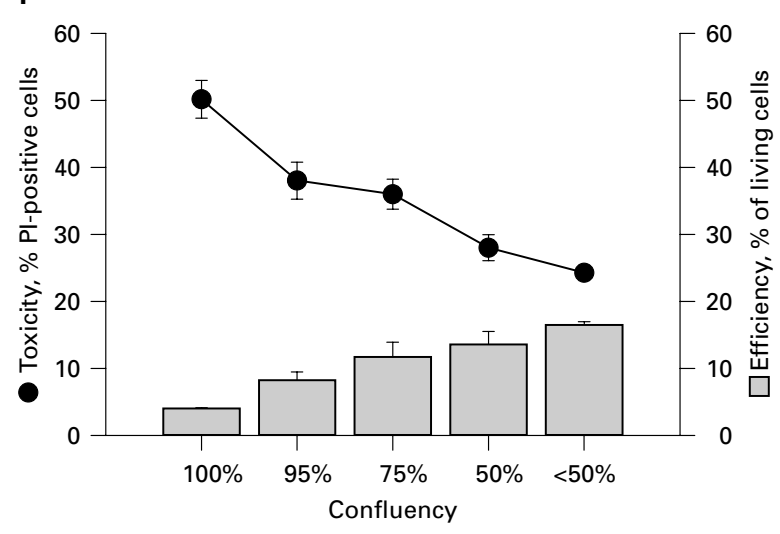

expression in \% of living, i.e. PI-positive cells, numbers in parentheses: of all cells). b-e Representative confocal images of GFP-expressing cells: MF + PEI 1,000 ng fluorescence (b), transmission (c), PEI 1,000 ng without MF fluorescence (d), and transmission (e). f Dependence of efficiency (bars) and toxicity (line) on the confluence state of HUVEC at the time of transfection. There was a linear correlation between toxicity (PI-positive cells) and increasing confluency and an inverted linear correlation between increasing confluency and efficiency of transfection $(n=4$ each). 
Fig. 4. $M F$ increases gene delivery into PAEC. Similar results as in HUVEC were found when MF was performed in PAEC (confluence $\sim 75 \%$ ). When using FuGENE $(\mathbf{b}, \mathrm{n}=3)$, PEI $(\mathbf{c}, \mathrm{n}=3)$, or DMRIETM $(\mathbf{a}, \mathrm{n}=3), \mathrm{MF}$ was able to increase luciferase activity severalfold. However, when using Lipofectamine (d, $\mathrm{n}=3$ ), neither MF nor conventional transfection resulted in any efficiency worth mentioning. Similar results were obtained when transfecting a $\beta$-galactosidase-coupled vector into PAEC. High efficiencies were achieved for PEI (e: 1,000 ng without MF, f: $1,000 \mathrm{ng}$ with MF) or FuGENE (g: 500 ng without MF, h: 500 ng with MF). Images representative of 6 independent experiments.
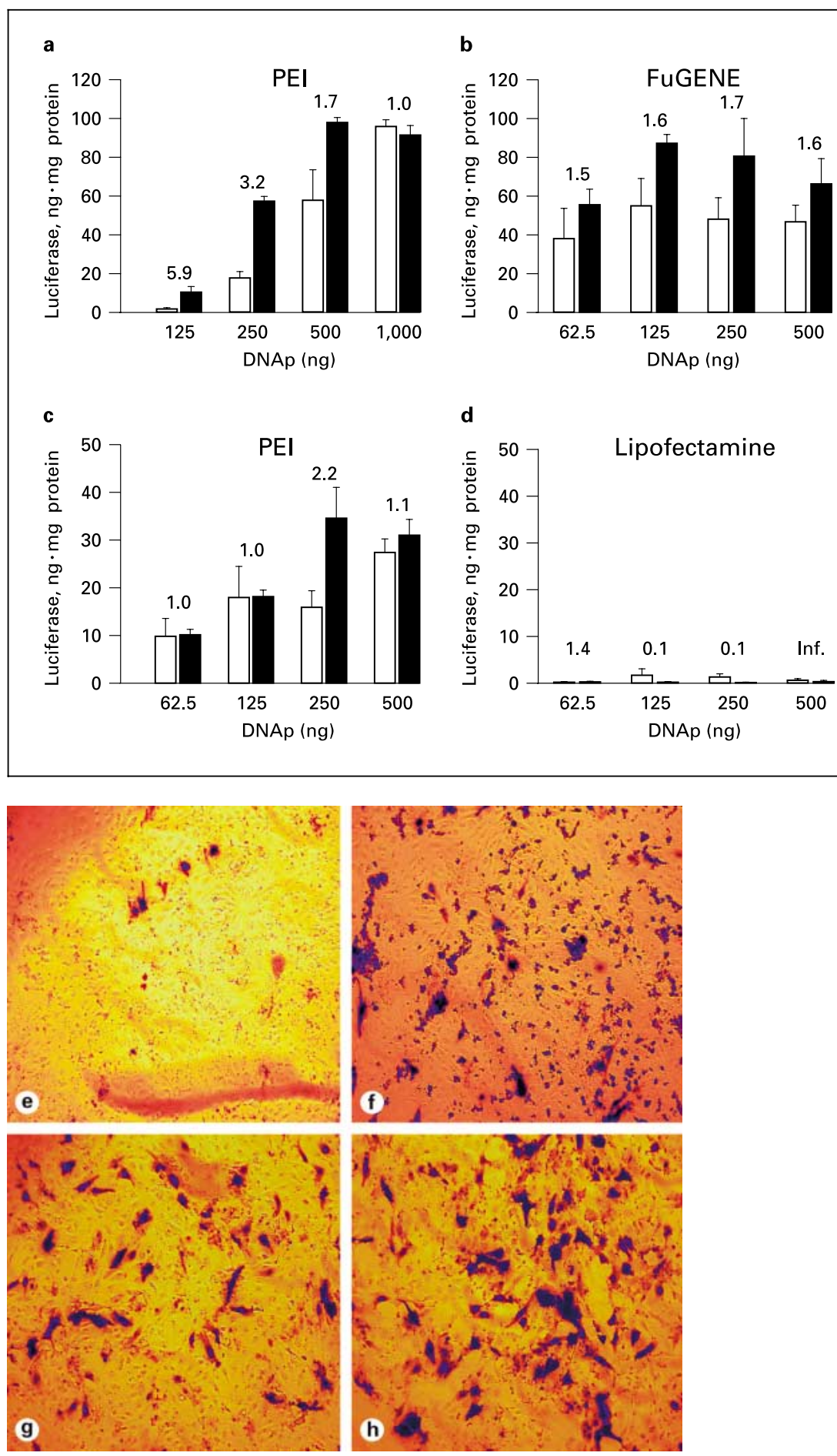

tivity to the toxicity of the procedure $(50.2 \pm 3 \%$ PI-positive cells). In general experiments revealed that the lower the confluency state of the cell layer was on the day of transfection the higher was the efficiency and the lower was the toxicity of the procedure (fig. 3f).

\section{Specific Cytotoxicity of MF}

To gain deeper insights into the specific agent that caused cytotoxicity after standard transfection or MF of plasmidic vectors, LDH release of HUVEC cultured on 96-well culture dishes (confluence state 90-100\%) was 
compared after conventional transfection of $\mathrm{p} 55 \mathrm{Luc}^{+}$using Lipofectamine and MF with Lipofectamine. The LDH activity in cells $24 \mathrm{~h}$ after exposure to the respective transfection reagent (as described in Methods) was compared to the LDH activity in the supernatants of nontransfected cells. For positive controls, cells were lysed by exposing them to Triton X-100 1\% for $10 \mathrm{~min}$, which led to $100 \%$ nonviable cells as confirmed by trypan blue staining. At all concentrations tested, MF significantly increased LDH activity when compared to exposure to the respective standard transfection (fig. 5). Assuming LDH release of untreated control cells to be $0 \%$ toxic and Triton treatment to be $100 \%$ toxic, control transfections using $62.5 \mathrm{ng} /$ well killed $18 \pm 10 \%$ of cells compared to $29 \pm 7 \%$ using MF. Therefore, at $62.5 \mathrm{ng} /$ well, MF caused a 1.6-fold increase in toxicity $(\mathrm{n}=11, \mathrm{p}<0.01)$. At $125 \mathrm{ng} /$ well this increase amounted to 2.3-fold $(\mathrm{n}=5, \mathrm{p}<$ $0.01)$, at $250 \mathrm{ng}$ it was 1.6 -fold $(\mathrm{n}=5, \mathrm{p}<0.01)$, and at $500 \mathrm{ng}$ it was equal to the standard transfection using Lipofectamine alone (MF: $35.6 \pm 0.8 \%$, standard: $37.9 \pm$ $0.2 \%, \mathrm{n}=3$ each, 0.9 -fold, nonsignificant). Although there were significant increases in cytotoxicity, this did not outweigh the advantageous efficiency caused by MF. When calculating the relative increases caused by MF and correcting the enhancement of luciferase expression for this increase in toxicity, the resulting corrected efficiency still revealed an up to 224-fold increase of luciferase activity in magnetofected HUVEC (table 1).

Comparing the toxicity of the Lipofectamine-luciferase transfection with the one caused by the PEI-GFP transfection (see above and fig. 3a-f) suggests that addition of the liposomal transfection reagent either/or the different plasmid, but not the amount of magnetic particles which remained the same, may have caused the diverging toxicity. To further investigate the actual component of the transfection mixture that was responsible for the toxic effect of the procedure, we continued to expose cells to different combinations of the single components with and without exposing them to a magnetic field. The results of these experiments (shown in fig. 5b) reveal that none of the single components alone, neither magnetic particles $(1 \mu \mathrm{g} /$ well) nor pure pDNA (500 ng/ well) nor both in combination with or without a magnetic field (experiments performed on 96-well plates), significantly increased basal LDH release of HUVEC. Apparently, the procedure only reached a toxic effect, when a transfection reagent (such as Lipofectamine in luciferase experiments or PEI in GFP experiments) was added so a successful transfection was reached (fig. 3f, 5a).

Magnetofection of pDNA to Endothelial Cells
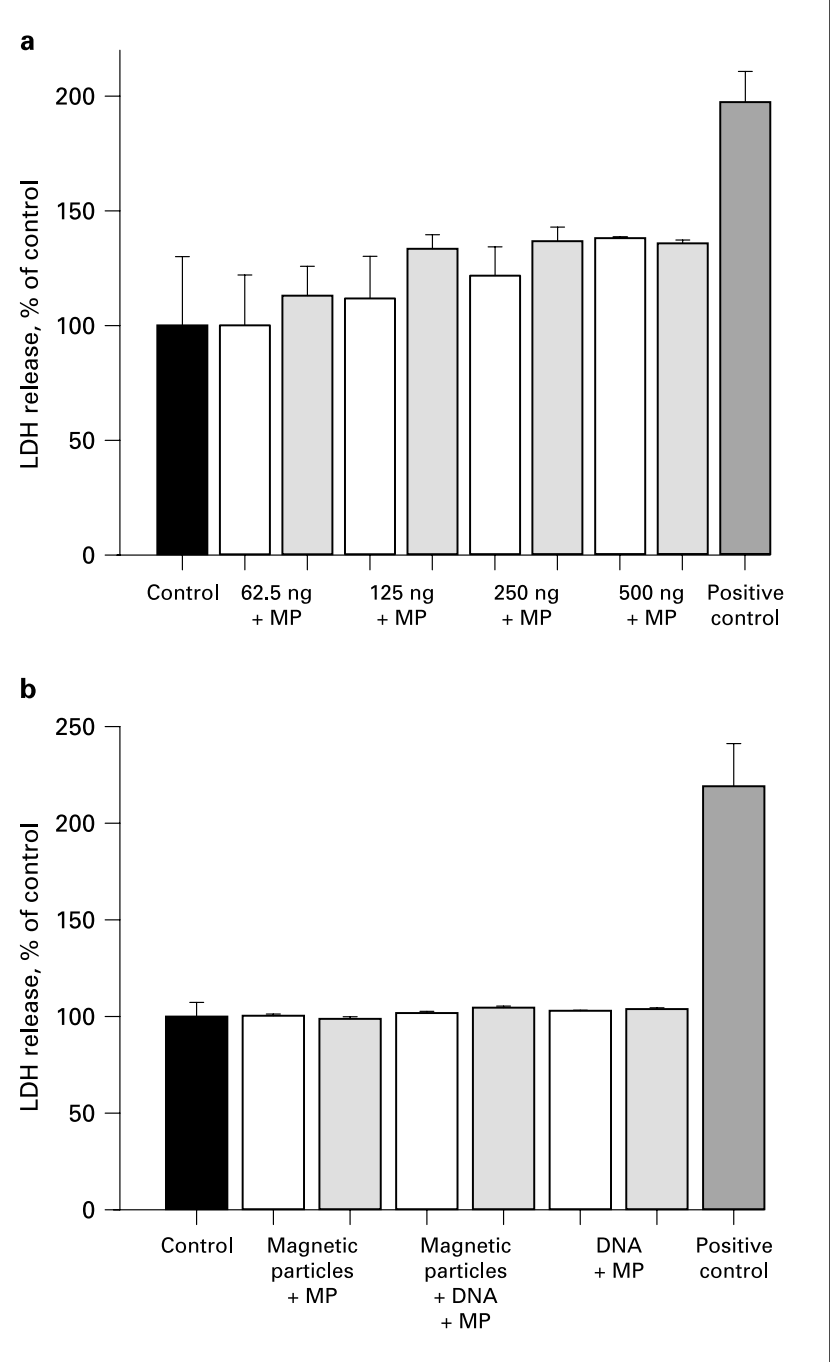

Fig. 5. Lactate dehydrogenase (LDH) activity of endothelial cells following MF. a Comparison of LDH activity released into the supernatants of p55 Luc ${ }^{+}$-transfected HUVEC (confluence 90-100\%) using Lipofectamine with (grey bars) or without (white bars) magnetic force. $\mathrm{LDH}$ release is expressed as $\%$ of the release of control cells (black bar). For positive controls all cells were killed using $10 \mathrm{~min}$ exposure to Triton X-100 1\% (dark grey bar, $n=5-11$ ). b LDH release of cells of similar confluency after $4 \mathrm{~h}$ of exposure to either magnetic particles, pDNA, or PDNA bound to magnetic particles, with or without exposure to a magnetic field (MF).

\section{Transfection of $p D N A$ into PAEC}

As in HUVEC, MF was equally able to increase the efficiency of transfection of a luciferase overexpressing plasmid in PAEC (confluence 60-80\%). The highest increase in luciferase activity by MF observed in these cells was 5.9-fold for $62.5 \mathrm{ng}$ pDNA using DMRIE, MF increased luciferase efficiency in any setup used, and

J Vasc Res 2003;40:425-434 431 
Table 2. Transfection efficiency of p55Luc ${ }^{+}$in HUVEC using Lipofectamine corrected for toxicity

\begin{tabular}{|c|c|c|c|c|c|}
\hline $\begin{array}{l}\text { pDNA } \\
\text { ng }\end{array}$ & $\begin{array}{l}\text { Increase in } \\
\text { efficiency achieved } \\
\text { by MF, n-fold }\end{array}$ & $\begin{array}{l}\text { Toxicity caused } \\
\text { by standard TF } \\
\text { LDH-release, } \\
\% \text { triton treatment }\end{array}$ & $\begin{array}{l}\text { Toxicity caused } \\
\text { by MF } \\
\text { LDH-release, } \\
\% \text { triton treatment }\end{array}$ & $\begin{array}{l}\text { Increase in } \\
\text { toxicity caused } \\
\text { by MF, n-fold } \\
\text { vs. standard TF }\end{array}$ & $\begin{array}{l}\text { Increase in } \\
\text { efficiency caused } \\
\text { by MF corrected } \\
\text { for toxicity, } n \text {-fold }\end{array}$ \\
\hline 62.5 & $\begin{array}{l}358.6 \\
\text { (control: } 0.4 \text { ng luciferase/ } \\
\text { mg protein) }\end{array}$ & $18 \pm 10$ & $29 \pm 8$ & 1.6 & 224.1 \\
\hline 125 & $\begin{array}{l}217.9 \\
\text { (control: } 2.2 \text { ng luciferase/ } \\
\text { mg protein) }\end{array}$ & $22 \pm 5$ & $49 \pm 6$ & 2.3 & 94.7 \\
\hline 250 & $\begin{array}{l}2.4 \\
\text { (control: } 50 \text { ng luciferase/ } \\
\text { mg protein) }\end{array}$ & $33 \pm 6$ & $53 \pm 6$ & 1.6 & 1.5 \\
\hline 500 & $\begin{array}{l}1.5 \\
\text { (control: } 8.9 \text { ng luciferase/ } \\
\text { mg protein) }\end{array}$ & $38 \pm 0$ & $36 \pm 1$ & 0.9 & 1.7 \\
\hline
\end{tabular}

All data describe $n$-fold increase caused by MF in comparison to the respective standard method (equalling $100 \%)$. Data representative of 5-11 experiments.

achieved the highest overall efficiency. However, using Lipofectamine, luciferase activity in PAEC could be not be transfected at all without MF in our experiments, and in this situation MF could not increase activity and therefore could also not lead to a satisfying result (all experiments $n=3$, for data see fig. $4 a-d$ ).

When transfecting a $\beta$-galactosidase-coupled vector into PAEC, MF again increased transfection efficiency. High efficiencies were achieved for PEI (fig. 4e, f) or FuGENE (fig. 4g, h), DMRIE yielded lower efficiencies. Table 2 shows the transfection efficiencies expressed as percent of cells positively staining for X-gal. In all setups, MF yielded increased transfection efficiency when compared to standard techniques, which reached a significance for 250 and $62.5 \mathrm{ng}$ of pDNA when using FuGENE, or 1,000 or $500 \mathrm{ng}$ of pDNA when using PEI ( $\mathrm{n}=6$ for all data). Again, 1,000 ng of PEI yielded maximum overall efficiency $(37.5 \pm 2 \%$ positively staining for $\mathrm{X}$-gal).

\section{Discussion}

In this study we show that MF enhances transfection efficiency of pDNA in endothelial cells, thought to be virtually transfection resistant, of two different species. Furthermore, MF allowed gene transfer into confluent cells, and, under certain conditions, allows transfection in combination with the cheap polycationic compound PEI, rather than in combination with expensive, commercially obtainable transfection mixtures. Although this is achieved at an increase in cellular cytotoxicity, the advantage of increased efficiency by far outweighs this enhanced cytotoxicity.

Among the primary cells targeted for gene delivery, cultured HUVEC or PAEC, both cell types that are conveniently used, are an interesting target for transfection with plasmid vectors, although they are relatively resistant to effective gene delivery [7-9]. For many years, acceptable efficiencies could only be reached by applying adenoviral constructs $[6,15]$. Recent data suggest, however, that optimized electroporation techniques can also achieve remarkable efficiencies of up to $40 \%$ [16]. Such levels of efficiency represent the highest documented transfection efficiencies for endothelial cells. However, electroporation as well as adenoviral transfection are limited by several problems. Electroporation requires expensive technical equipment and exerts high rates of cytotoxicity of $60 \%$ of cells or more $[16,17]$. Adenoviral gene delivery, on the other hand, is restricted by a need for strict security measures. Furthermore, interpretation of experimentally achieved results is difficult because any observed effect might be caused by a simultaneous cellular expression of unknown viral proteins. For such reasons, nonviral, liposomal techniques for gene delivery into primary cells have been intensely investigated [19]. We show here that MF offers a simple, effective and cheap improvement of plas- 
midic gene delivery that results in an up to several hundredfold increased efficiency in target gene expression in HUVEC and PAEC. This increase in efficiency is accompanied by a significant increase of cellular toxicity caused by the magnet-based protocol. However, magnetic particles or exposure of cells to magnetic fields do not appear to cause this toxicity according to our data. Only transfection mixtures containing all compounds needed to achieve satisfactory results (reagent, such as PEI or a liposomal compound, pDNA, and magnetic nanobeads) had toxic effects on cultured endothelial cells, suggesting that either the addition of the transfection reagent or the integration procedure of transfected pDNA into cellular nuclei and the resulting protein expression take part in a cytotoxic effect. Furthermore, we used a very sensitive mode of assessment of toxicity. As control cells are assumed to all be viable ( 0 toxicity) in our data, the results obtained are likely to rather overestimate the toxicity of the procedure. We have previously shown that using MF high efficiency is already reached after $15 \mathrm{~min}$ and that efficient short-term MF of oligonucleotides results in a decreased cytotoxicity in HUVEC [11, 12]. However, in order to compare toxicity and efficiency of MF with standard transfections, MF and control mixtures containing the toxic cationic lipid reagents remained on cells for a full $4 \mathrm{~h}$ in most experiments demonstrated here. After removal of the magnet, this appeared to be the necessary time to achieve satisfying results using the standard method. Considering that the effective amount of the transfection reagent mixture directly covering the cells was probably far enhanced in the MF group, an increased toxicity caused by the increased lipid reagent concentration on the cell surfaces seems likely. Therefore, the toxicity of the method is probably even further overestimated. Nevertheless, when adjusting the increase in toxicity to the increase in efficiency, MF still exceeded the effectiveness achieved by conventional cationic transfection several hundred-fold.

Dose-response curves for increasing concentrations of e.g. the luciferase plasmid used in our experiments do not behave according to a linear correlation. In many cases, there is rather a peak at one certain concentration, from which on efficiency cannot be further enhanced by increasing pDNA doses, which is probably due to prevailing toxicity. This rising toxicity is caused by the use of higher amounts of transfection reagent for higher doses of pDNA. Therefore, dose-response curves obtained for one transfection reagent or plasmid may not simply be conferred to another one, as shown by the different results depending on transfection reagent using a luciferase plas- mid. Thus, this study outlines the importance of performing extensive dose-response tests before using a reagent with a special plasmid to transfect endothelial cells.

Although the transfection efficiency showed great divergence depending on the transfection reagents used and the confluence state of the HUVEC, MF significantly improved transfection success in 34 out of 40 transfection protocols in our study. FuGENE and simple complexing of pDNA with PEI without further use of a liposomal reagent were most effective in nonconfluent cells, but only $\mathrm{PEI}$, a small cationic molecule, in combination with $\mathrm{MF}$ was similarly successful for confluent cells. However, the confluency state of cells remains an important aspect for planning efficient plasmid transfection, as we could observe that not only did the efficiency of MF decrease, but also the toxicity of the procedure rose with increasing confluency of endothelial cell layers. It remains to be mentioned, however, that efficiency in terms of percent of cells expressing the transfected protein or enzyme, but also the level of activity of this target is a desirable target. Especially in transfections of a luciferase-encoding plasmid, where assessment of the success of the procedures is not expressed as percent of transfected cells, but rather as activity of the transfected enzyme, lower percentages of expressing cells may still reveal high enzymatic activity. MF furthermore succeeded in lowering the amount of DNA needed to achieve an efficient procedure in 9 out of 12 applications of different combinations of transfection reagent with cell type.

The mechanism of cellular uptake of the magnetic bead-coupled pDNAs still remains to be revealed. The increased effectiveness could simply be due to an increased effective concentration of pDNA at the outer cellular membrane. In vitro, an increase of an effective concentration at the outer cellular membrane could perhaps also be reached by other means such as increasing pDNA concentrations or by centrifugation of transfection complexes onto cells. However, we have previously observed that centrifugation of pDNA onto several cell lines cannot equal the effect gained by MF [11], and increasing pDNA and with it the concentrations of the transfection reagent goes along with enhanced cytotoxicity. Notably, centrifugation is also not applicable in vivo.

The advantages of MF and the finding that it is an easy and effective technique to transfect endothelial cells give rise to expectations as to its possible application in vivo. The promising scenario of exerting a magnetic field only to specific sites of an organism, thereby targeting plasmid vectors to this site, is nourished by the short time needed for effective transfection, as previously shown in certain 
cell lines [11] or endothelial cells, which altogether increases the likeliness of a successful therapeutic intervention. Especially in cardiovascular therapy enormous efforts have been put into such site-directed therapy [5, 20, 21]. For example, a catheter-based coronary angioplastic therapy, which has emerged as state of the art treatment for occlusive cardiovascular disease [3, 21], could be effectively supplemented by MF. Similar approaches could be used for peripheral vascular disease, which is already the subject of clinical gene therapy studies.

However, a number of points remain unresolved and will be the subject of future investigations. These include the question of whether a formulation of transfection reagents can be found that minimizes the immunologic responses caused by all commonly used cationic liposo- mal transfection reagents. One key towards a solution of such problems might be the finding that only in combination with PEI, a simple small cationic molecule, efficient expression of luciferase activity could be reached in confluent HUVEC. In this context, it is notable that the mean activity reached in these experiments exceeded the maximum activity reached by any formulation in nonconfluent cells.

In summary, the feasibility and high effectiveness of magnet-based transfection of endothelial cells should allow simple application and important advantages in experimental cellular investigation, and might be an important step in enhancing the likeliness of reasonable in vivo vascular gene transfer, which so far is not at hand.

\section{References}

1 Haines AM, Irvine AS, Mountain A, et al: CL22 - A novel cationic peptide for efficient transfection of mammalian cells. Gene Ther 2001;8:99-110.

2 Gottschalk S, Sparrow JT, Hauer J, et al: A novel DNA-peptide complex for efficient gene transfer and expression in mammalian cells. Gene Ther 1996;3:48-57.

3 Ross R: The pathogenesis of atherosclerosis: A perspective for the 1990s. Nature 1993;362: 801-809.

4 Yla-Herttuala S, Martin JF: Cardiovascular gene therapy. Lancet 2000;355:213-222.

5 Taniyama Y, Tachibana K, Hiraoka K, et al: Local delivery of plasmid DNA into rat carotid artery using ultrasound. Circulation 2002;105: 1233-1239.

6 Modlich U, Pugh CW, Bicknell R: Increasing endothelial cell specific expression by the use of heterologous hypoxic and cytokine-inducible enhancers. Gene Ther 2000;7:896-902.

7 Edgell CJ, Curiel DT, Hu PC, Marr HS: Efficient gene transfer to human endothelial cells using DNA complexed to adenovirus particles Biotechniques 1998;25:264-266.

8 Tanner FC, Carr DP, Nabel GJ, Nabel EG: Transfection of human endothelial cells. Cardiovasc Res 1997;35:522-528.
9 Teifel M, Heine LT, Milbredt S, Friedl P: Optimization of transfection of human endothelial cells. Endothelium 1997;5:21-35.

10 Colombo MG, Citti L, Basta G, De Caterina R, Biagini A, Rainaldi G: Differential ability of human endothelial cells to internalize and express exogenous DNA. Cardiovasc Drugs Ther 2001;15:25-29.

11 Scherer F, Anton M, Schillinger U, et al: Magnetofection: Enhancing and targeting gene delivery by magnetic force in vitro and in vivo. Gene Ther 2002;9:102-109.

12 Krötz F, de Wit C, Sohn H-Y, et al: Magnetofection, a highly efficient tool for antisense oligonucleotide delivery in vitro and in vivo. Mol Ther 2003;7:700-710.

13 Krötz F, Sohn H-Y, Keller M, et al: Depolarization of endothelial cells enhances platelet aggregation through oxidative inactivation of endothelial NTPDase. Arterioscler Thromb Vasc Biol 2002;22:2003-2009.

14 Gloe T, Riedmayr S, Sohn HY, Pohl U: The $67-\mathrm{kDa}$ laminin-binding protein is involved in shear stress-dependent endothelial nitric-oxide synthase expression. J Biol Chem 1999;274: 15996-16002.
15 Hay CM, De Leon H, Jafari JD, et al: Enhanced gene transfer to rabbit jugular veins by an adenovirus containing a cyclic RGD motif in the HI loop of the fiber knob. J Vasc Res 2001;38:315-323.

16 Ear T, Giguere P, Fleury A, Stankova J, Payet MD, Dupuis G: High efficiency transient transfection of genes in human umbilical vein endothelial cells by electroporation. J Immunol Methods 2001;257:41-49.

17 Schwachtgen JL, Ferreira V, Meyer D, Kerbiriou-Nabias D: Optimization of the transfection of human endothelial cells by electroporation. Biotechniques 1994;17:882-887.

18 Lewis E, Rudo T, St John MR, et al: Endothelial cell DNA transfer and expression using petri dish electroporation and the nonreplicating vaccinia virus/T7 RNA polymerase hybrid system. Gene Ther 1999;6:1617-1625.

19 Kaiser S, Toborek M: Liposome-mediated high-efficiency transfection of human endothelial cells. J Vasc Res 2001;38:133-143.

20 Morishita R, Aoki M, Kaneda Y, Ogihara T: Gene therapy in vascular medicine: Recent advances and future perspectives. Pharmacol Ther 2001;91:105-114.

21 Landau C, Lange RA, Hillis LD: Percutaneous transluminal coronary angioplasty. N Engl J Med 1994;330:981-993. 